Cahiers de recherches médiévales

\title{
Le prince et le félon
}

Le siège de Guinesores dans le Cligès de Chrétien et dans la prose bourguignonne

\section{Michelle Szkilnik}

\section{(2) OpenEdition}

Édition électronique

URL : https://journals.openedition.org/crm/2654

DOI : $10.4000 / \mathrm{crm} .2654$

ISSN : 1955-2424

Éditeur

Honoré Champion

\section{Édition imprimée}

Date de publication : 15 décembre 2007

Pagination : 61-74

ISSN : 1272-9752

\section{Référence électronique}

Michelle Szkilnik, «Le prince et le félon », Cahiers de recherches médiévales [En ligne], 14 | 2007, mis en ligne le 15 décembre 2010, consulté le 15 décembre 2022. URL : http://journals.openedition.org/crm/ 2654 ; DOI : https://doi.org/10.4000/crm.2654 


\section{酮RM}

\section{Le prince et le félon : \\ Le siège de Guinesores dans le Cligès de Chrétien et dans la prose bourguignonne ${ }^{1}$}

Lorsque le jeune Alexandre de Constantinople, futur père de Cligès, se rend pour la première fois à la cour d'Arthur, il est reçu par le roi en majesté : «couronné et seant en son siege royal» (p.70). Tel est l'un des détails dont le Cligès bourguignon enrichit la description de l'entrevue donnée par Chrétien de Troyes². Simple détail, certes, mais significatif: le roi Arthur des mises en prose du $\mathrm{XV}^{\mathrm{e}}$ siècle est un prince imposant, à l'image du duc de Bourgogne à la cour de qui des écrivains anonymes ont "transmué de rime en prose» (p. 164) l'œuvre de Chrétien.

Les mises en prose bourguignonnes de Cligès et d'Erec ont récemment fait l'objet de stimulantes études qui permettent d'apprécier à leur juste valeur les modifications apportées aux romans du XII ${ }^{\mathrm{e}}$ siècle $^{3}$. Les éditions que Maria Colombo Timelli vient de procurer encouragent par ailleurs de nouvelles recherches dans ce domaine $^{4}$. C'est dans les perspectives ouvertes par mes prédécesseurs que je voudrais inscrire cette contribution consacrée à un épisode de Cligès: le siège de Guinesores (Windsor) par les troupes d'Arthur, à l'occasion duquel se dessinent les figures opposées du traître et du souverain.

\footnotetext{
1 Toutes mes références au Cligès en prose sont empruntées à la récente édition de M. Colombo Timelli : Le Livre de Alixandre empereur de Constentinoble et de Cligés son fils, Genève, Droz, 2004. Pour le texte de Chrétien, j'utilise l'édition d'O. Collet et C. Méla, Paris, Livre de poche (Lettres Gothiques), 1994. Cette édition se fonde sur le ms C (BNF fr 12560), représentant de l'une des deux grandes familles de manuscrits du Cligès, la famille $\gamma$. Or ce manuscrit figurait dans la bibliothèque des Ducs de Bourgogne. M. Colombo Timelli a certes relevé quelques différences entre la copie $\mathrm{C}$ du roman de Chrétien et la mise en prose, mais ne suggère pas moins «un raport de la prose avec un manuscrit de la famille $\gamma$ » (intro. à son édition p. 47-49 pour la discussion d'ensemble).

${ }^{2}$ Chrétien se contente de dire qu'Arthur était « a sejor » à Guincestre (v. 303).

${ }^{3}$ Voir J. H.M. Taylor, "The Significance of the Insignificant: Reading Reception of the Burgundian Erec and Cligès », Fifteenth-Century Studies, XXIV, 1998, p. 183-97 ; N. J. Lacy, "Motivation and Method in the Burgundian Erec», Conjunctures: Medieval Studies in Honor of Douglas Kelly, éd. K. Busby et N. J. Lacy, Amsterdam, Rodopi, 1994, p. 271-80, et "Adaptation as Reception: The Burgundian Cligès», Fifteenth-Century Studies, XXIV, 1998, p. 198-207 ; parmi les nombreux articles de M. Colombo Timelli, citons en particulier «Le Cligès en prose (1455) ou l'actualisation d'un ancien conte en vers», L'analisi linguistica e letteraria, 1-2, 2000, p. 329-42 ; voir aussi C. Descheper, « De l'adultère comme résistance à l'empereur usurpateur, la convergence des intrigues amoureuses et politiques dans le Cligès en prose ", La Littérature à la cour de Bourgogne, Actualités et perspectives de recherches, éd. C. Thiry et T. Van Hemelryck, Le Moyen Français, 57-58, 2006, p. 67-86.

${ }^{4}$ Voir note 1 pour Cligès; pour Erec: L'histoire d'Erec en prose, roman du XV siècle, Genève, Droz, 2000.
}

Cahiers de Recherches Médiévales, 14, 2007 
$\mathrm{Au}$ début du roman, le roi se rend en Bretagne et en son absence confie son royaume au comte Engrès. Mais, précurseur de Mordret, celui-ci trahit la confiance d'Arthur et le roi est obligé de revenir précipitamment en Angleterre. L'aventure guerrière se déroule en parallèle avec l'aventure amoureuse d'Alexandre, fils aîné de l'empereur de Constantinople venu se faire adouber à la cour arthurienne, et la jeune Soredamor, sœur de Gauvain. L'épisode occupe chez Chrétien environ 1200 vers sur un total de 6702 vers $^{5}$. Il représente donc plus d'un sixième du roman. Dans la version en prose, il s'étend de la page 79 à la page 98 , dix-neuf pages sur un total de cent, soit un cinquième du roman. C'est dire que l'un et l'autre textes accordent une importance remarquable à l'épisode. Comme on sait que les mises en prose ont tendance à abréger leur source, il est notable que dans ce cas précis, le prosateur bourguignon augmente légèrement la proportion réservée à l'aventure ${ }^{6}$. Ces chiffres dissimulent cependant une distorsion bien connue par ailleurs: les prosateurs tendent à couper les développements sur l'amour mais à amplifier au contraire tout ce qui relève des prouesses chevaleresques. Or, on l'a dit, la trahison du comte Engrès sert de toile de fond à la naissance de l'amour entre Alexandre et Soredamor, ou, plus exactement, Chrétien entrelace habilement le récit de l'expédition punitive et la description des émois amoureux des deux jeunes gens, maintenant un équilibre savant entre les deux pôles de l'épisode. Si le prosateur déroule l'histoire de la même manière que Chrétien, il contracte néanmoins sensiblement la part réservée à l'aventure amoureuse.

Examinons la structure du passage pour mesurer d'un point de vue purement quantitatif d'abord la différence d'équilibre entre le texte source et la mise en prose?

1. Annonce de la trahison. Conseil de guerre. Arthur rassemble son armée pour repasser en Grande Bretagne. Alexandre vient demander au roi qu'il le fasse chevalier en compagnie des autres jeunes Grecs. (Chrétien: v. 1049-140, prose: p. 79-81, 42 lignes).

2. La reine remet à Alexandre la chemise confectionnée par Soredamor, dans laquelle la jeune fille a cousu l'un de ses cheveux d'or. Eloge d'Alexandre dont la beauté surpasse celle de tous les autres jeunes Grecs (Chrétien v. 1141-202, prose : p. 81, 13 lignes).

3. Fuite d'Engrès qui se réfugie à Guinesores. Débarquement d'Arthur. Description de la forteresse qu'il va assiéger. Escarmouche sous les murs de Guinesores entre Alexandre et ses compagnons et des hommes d'Engrès. Alexandre

\footnotetext{
${ }^{5}$ Du vers 1049 : l'annonce de la trahison, au vers 2210 : Soredamor aprend qu'Alexandre est vivant et qu'il a remporté la coupe promise par Arthur à celui qui permettra la prise de Guinesores.

${ }^{6}$ Notons encore que sur les neuf espaces blancs réservés sans doute à des miniatures dans le manuscrit unique du Cligès en prose, trois se situent dans l'épisode de Guinesores, témoignant ainsi de l'importance que lui accorde le prosateur (voir M. Colombo Timelli, intro. à son édition, p. 20).

${ }^{7}$ Les chiffres indiqués sont approximatifs. Il est en effet artificiel de découper les deux romans et certains passages de transition pourraient être rattachés à l'une ou l'autre des intrigues. C'est la proportion qu'ils révèlent qui est importante. Par ailleurs le résumé proposé se fonde sur la version de Chrétien. Or, on le verra, certains détails disparaissent de la version en prose qui en revanche en ajoute d'autres.
} 
en capture quatre qu'il remet à la reine. Mécontentement du roi qui mande la reine afin d'obtenir qu'elle lui remette les prisonniers (Chrétien v.1203-363, prose : p. 8184, 90 lignes).

4. Alexandre dans la tente des jeunes filles. Soredamor aperçoit le cheveu cousu dans la chemise et se demande comment aborder le jeune homme dont elle est amoureuse (Chrétien v.1364-410, prose : p. 84-85, 25 lignes).

5. Le sort des quatre prisonniers est réglé. Assaut de la forteresse arrêté par la nuit. Le roi promet une coupe d'or à celui qui prendra Guinesores (Chrétien v. 1411544, prose p. 85-87, 64 lignes).

6. Alexandre dans la chambre de la reine. Celle-ci remarque le cheveu d'or et fait avouer à Soredamor que c'est celui de la jeune fille. Joie extrême d'Alexandre (Chrétien v. 1545-636, prose : p. 88-89, 31 lignes).

7. Tentative de sortie des assiégés. Riposte des assiégeants. Alexandre et ses compagnons pénètrent par ruse dans la forteresse et la conquièrent. Deuil des Grecs qui, ayant trouvé les armes abandonnées par Alexandre, pensent qu'il est mort (Chrétien v. 1637-2075, prose p. 89-97, 196 lignes).

8. Lamentations de Soredamor sur la mort d'Alexandre (Chrétien v. 2076-88, prose : aucune mention de la douleur de Soredamor).

9. Deuil dans le camp d'Arthur. Alexandre envoie ses prisonniers se rendre à Arthur. La joie éclate dans le camp. Arthur remet la coupe d'or à Alexandre et promet de lui donner tout ce qu'il peut désirer. Alexandre offre la coupe à Gauvain. (Chrétien : v. 2089-199, prose p. 96-98, 93 lignes).

10. Soredamor apprend avec joie qu'Alexandre est vivant (Chrétien v. 220010, prose : p. 98, 6 lignes).

La prose consacre donc 75 lignes à l'aventure amoureuse et 485 à l'entreprise guerrière, soit, grossièrement, une proportion de 1 pour 6 ; avec 220 vers pour l'amour et 632 pour les exploits chevaleresques, Chrétien offre une proportion, très approximative, de 1 pour 3 . Si globalement l'épisode de la trahison occupe une place plus grande par rapport à l'ensemble du roman en prose, seul le pôle guerrier bénéficie de l'amplification constatée 8 .

Je ne m'attarderai guère sur la manière dont la prose abrège l'intrigue amoureuse: ce phénomène a déjà été bien étudiée. La réduction de l'intrigue amoureuse, paradoxale quand il s'agit d'adapter un roman de Chrétien, correspond à un certain désintérêt pour la peinture de l'amour caractéristique de la littérature romanesque tardive. Le prosateur va donc atténuer ou ignorer les émotions des personnages. Ainsi alors que Chrétien imagine les réactions que ressentiraient les deux amoureux s'ils savaient que le jeune homme porte la chemise cousue par la

\footnotetext{
${ }^{8}$ Il est difficile d'établir une correspondance exacte entre le texte de Chrétien et le texte en prose dans la mesure où les innovations introduites par ce dernier se concentrent justement dans le récit du siège et des combats. En d'autres termes, les divergences sont parfois telles, on va le voir, qu'il ne s'agit plus à proprement parler d'amplification ou d'abrègement, mais bien de l'introduction de scènes nouvelles.

${ }^{9}$ Voir N.J. Lacy, «Adaptation as Reception : the Burgundian Cligès », art. cit., en particulier p. 200-02 ; M. Colombo Timelli, intro. à son éd. p. 31-32.
} 
jeune fille ${ }^{10}$, la prose n'en souffle mot. De même, le long monologue de Soredamor sur le doux nom d'ami qu'elle n'ose donner à Alexandre est considérablement raccourci. Les exclamations, les questions, le dédoublement du personnage, bref, toute la rhétorique précieuse disparaissent au profit d'un discours plus simple et plus explicite. En voici un exemple frappant:

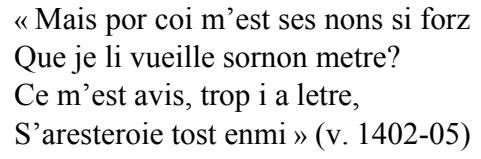

devient: «Son nom Alixandre est long a proferer quant a une pucelle» (p. 85). Comme je l'ai noté dans mon résumé, la prose ne rapporte pas la douleur de Soredamor à l'annonce de la mort supposée d'Alexandre, alors qu'en revanche, elle note le chagrin de Gauvain et des chevaliers de la Table Ronde. Par ailleurs, le prosateur tend à ajouter des détails qui rationalisent ou atténuent l'audace de certains comportements. Quand Alexandre découvre le cheveu d'or de Soredamor dans la chemise qu'il porte, il est ivre de joie. Chrétien se plaît à décrire avec une certaine malice les réactions du jeune homme qui n'ose d'abord baiser la chemise devant la reine mais, sitôt rentré chez lui, s'abandonne à des transports fétichistes qui font dire à Chrétien : «Bien fet Amors de sage fol/Quant cil fet joie d'un chevol» (v. 163334). Si la prose conserve le nombre de cent mille baisers dont Alexandre couvre la chemise, elle abrège considérablement le récit de ses démonstrations et omet la sentence de Chrétien, pressée qu'elle est d'en revenir au sort des traîtres capturés par Alexandre, que la scène du cheveu d'or a différé. Elle apporte toutefois une précision concernant l'attitude de Soredamor: sommée d'avouer qu'elle a cousu l'un de ses cheveux dans la chemise, la jeune fille, dans les deux versions, rougit de pudeur mais, chez Chrétien, elle est bien contente de reconnaître que le cheveu lui appartient. Dans la prose, elle le confesse avec plus de réticence et atténue la portée de son acte : «elle s'en excuse disant que ce fust par mesadventure ou mespresure » (p. 88), mensonge qui a peut-être pour but de préserver la modestie attendue de toute jeune fille bien élevée.

Beaucoup plus intéressantes, dans la perspective qui est la mienne, sont les modifications que le prosateur fait subir à l'intrigue guerrière. Certaines ont déjà été relevées et commentées par d'autres avant moi $^{11}$. Je me propose toutefois de les reprendre d'ensemble pour montrer comment elles promeuvent l'image d'un chef de guerre, un chevetaine, dont les traits correspondent à ceux que l'on rencontre dans des textes contemporains. En m'appuyant sur les travaux de Maria Colombo

\footnotetext{
${ }^{10}$ Voir v. 1165-77 («Et Dex ! Com grant joie en eüst /Alixandres se il seüst/ Que la reïne li envoie !/ Molt en reüst cele grant joie/ Qui son chevol i avoit mis...»).

${ }^{11}$ En particulier M. Colombo Timelli dans l'introduction à son édition p. 32-34 et dans l'article «Le Cligès en prose (1455) ou l'actualisation d'un ancien conte en vers", art.cit. Voir aussi C. C. Willard, "The Misfortunes of Cligès at the Court of Burgundy », Arturus Rex, Acta Conventus Lovaniensis 1987, Leuven: Leuven University Press, 1991, vol. II, p. 397-403.
} 
Timelli $^{12}$ et le concept $d$ ' «acculturation» emprunté à Jane Taylor ${ }^{13}$, je voudrais mettre en évidence la cohérence du travail de réécriture dans tout l'épisode. Ce qui est tout à fait remarquable en effet, c'est d'une part la manière dont la prose recentre l'épisode autour du personnage d'Alexandre, d'autre part comment à cette occasion, elle projette une image du pouvoir plus conforme à la conception que s'en fait le public de la cour de Bourgogne. Le prosateur «corrige» par petites touches le portrait d'Alexandre ainsi que celui d'Arthur afin de les mettre en harmonie avec la réalité idéologique du $\mathrm{XV}^{\mathrm{e}}$ siècle.

Alexandre est évidemment le héros incontesté du siège et des batailles autour de Guinesores dans les deux versions. Toutefois le roman en prose accentue singulièrement cet aspect, peut-être plus diffus chez Chrétien. Un détail est de ce point de vue significatif: les jeunes Grecs, compagnons d'Alexandre, dont Chrétien énumère les noms à l'occasion de la première escarmouche sous les remparts de Guinesores $^{14}$, perdent leur identité. Ils se fondent en un groupe sans visage, celui des «chevaliers» d'Alexandre, sa «mesnie $»^{15}$, «ses gens de Grece» (p. 80), ses «hommes ». Alors que le texte en vers en distingue quelques-uns durant les combats (Calcedor, tué par le comte Engrès, Nabunal le sage), la prose ne les mentionne pas. La solidarité des jeunes Grecs se manifeste aussi à l'occasion de leur adoubement. L'Alexandre de Chrétien se fait le porte-parole de ses compagnons pour demander au roi Arthur qu'il les adoube tous les douze :

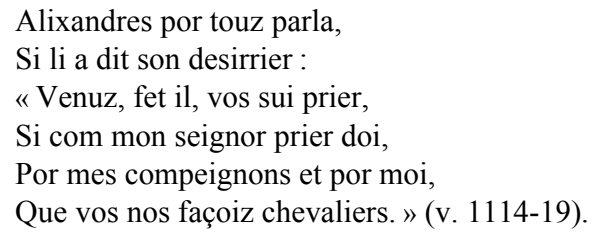

L'Alexandre du texte en prose ne demande l'adoubement que pour lui-

«Sire, comme il soit ainsi que je soie venu en ton service pour aprendre et obtenir de ta main le degré de chevalerie, je te pri qu'il te plaise par ta grace moy adouber se je

\footnotetext{
${ }^{12}$ «Le Cligès en prose (1455) ou l'actualisation d'un ancien conte en vers », art. cit., voir en particulier p. 339-41.

${ }_{13}$ «A process whereby the socio-culturally unfamiliar is recast in familiar terms, so that the reader can understand systems and phenomena in a source text as corresponding to his own ideologies, preconceptions, and behavior patterns. » («The Significance of the Insignificant : Reading Reception of the Burgundian Erec and Cligès », art. cit., p. 183).

${ }^{14}$ «S'en apele ses compeignons /L'un aprés l'autre par lor nons,/ Premier Cornin qu'il ama molt,/ Et puis Licoridés l'estout/ Et puis Nabunal de Micenes/ Et Acarionde d'Athenes/ Et Ferulin de Salenique, /Et Calcedor devers Aufrique,/ Permenidos et Francagel,/Coron le fort et Pinagel, Nerius et Neriolis. » v. 1273-83. Voir aussi v. 2039-41 : les noms de quelques-uns des compagnons d'Alexandre sont cités au moment où ils retrouvent les armes d'Alexandre et se livrent au désespoir.

${ }^{15}$ Le terme est employé p. 80 et p. 84 .
} 
suis propice ad ce, et Dieux doint que ce soit a mon honneur et a ton proffit. » (p. 80).

Le prosateur suit certes le plan général des opérations tel que l'a conçu Chrétien: premier affrontement sous les remparts, tentative de sortie, combats violents devant la forteresse, ruse d'Alexandre qui pénètre dans le château en compagnie de plusieurs chevaliers, capture du comte. La mise en œuvre de chacune des phases s'avère toutefois très différente dans le détail. Ainsi, lorsque les assiégés tentent une sortie de nuit mais sont aperçus par le guet, le texte en vers décrit d'abord longuement la contre-attaque des «royaux», puis s'attarde sur les Grecs (Alexandre en particulier mais ses compagnons également), avant de louer de nouveau la vigueur de l'armée royale. Après avoir parlé de ceulx de Bretaigne (p. 90), la version en prose en vient très vite à Alexandre, le premier sur les rangs car «a ceste premiere envahie n'est pas le roy Artus ne la plus part de sa chevalerie» (p. 90). Alors que Chrétien oppose au héros des adversaires anonymes qu'Alexandre coupe en morceaux avec enthousiasme, opération qui est décrite avec un certain humour $^{16}$, le prosateur imagine un combat singulier contre le sénéchal du comte Engrès «qui fait grant proesse de chevalerie» (p. 91). S'élançant au milieu de plus de cinq cents ennemis, Alexandre frappe de droite et de gauche pour parvenir au sénéchal. Le combat entre les deux hommes se déroule selon un patron épique familier: le premier coup d'Alexandre "genchist», le sénéchal riposte, mais Alexandre se protège habilement de son écu et frappe un second coup qui coupe en deux l'adversaire. Les archers ennemis se précipitent alors sur lui, il est désarçonné et continue à se battre vaillamment à pied. Gauvain et quelques compagnons viennent à la rescousse et le remontent sur un cheval. Les deux bons chevaliers se livrent ensuite de concert à un carnage. Le combat contre le sénéchal prépare celui qui opposera Alexandre à Engrès lui-même. Quant à l'entrée en scène de Gauvain qui ne joue pas de rôle particulier dans l'épisode chez Chrétien, elle permet d'affirmer le statut exceptionnel déjà atteint par le jeune Grec: il est l'égal de Gauvain, la fleur de la chevalerie arthurienne. Et par ailleurs, elle souligne l'importance du compagnonnage chevaleresque, valeur déjà prisée à l'époque de Chrétien (on songe à l'amitié qui unit Gauvain et Yvain dans Le Chevalier au lion), mais peut-être plus fortement louée encore dans la littérature du $\mathrm{XV}^{\mathrm{e}}$ siècle $^{17}$.

D'autres modifications apportées par le prosateur vont dans le même sens. La ruse imaginée par Alexandre pour pénétrer dans la forteresse est présentée de manière sensiblement différente d'un texte à l'autre. Chez Chrétien, Alexandre demande à quelques chevaliers qui le suivaient de loin (six Grecs et vingt-quatre Gallois) de bien vouloir rentrer dans son plan :

\footnotetext{
${ }^{16}$ Voir v. 1741-43 par exemple : «Quant a celui ot trive prise, / A .I. autre offre son servise / Ou pas ne le gaste ne pert. »

${ }^{17}$ On peut ainsi évoquer quelques «couples» de chevaliers : Saintré et Boucicaut dans Jean de Saintré, le duc de Clèves et Jacques dans Le Livre des faits de messire Jacques de Lalaing. Peut-être faut-il voir dans la célébration de cette valeur à la fois une influence de la littérature épique et un reflet de la réalité sociologique.
} 
« Seignor, fet il, sanz contredit,

Se vos volez m'amor avoir,

Ou face folie ou savoir,

Creantez moi ma volenté » (v. 1800-04).

Ceux-ci ayant donné leur accord, Alexandre leur expose son audacieuse idée et tous s'y rangent. Dans la version en prose, Alexandre ne parle pas à des chevaliers d'un rang égal au sien et dont il cherche à obtenir le consentement, mais à ses hommes dont il attend l'obéissance :

«Il appelle une Xe de sez honmes, et leur fait mettre jus leurz escus et prendre ceulx de leurz ennemis qui gesoient a terre, et mesmes, aprés ce, prendent il leur chevaulx ; » (p. 92).

Ce n'est qu'ensuite qu'il leur explique ce qu'il entend faire et il termine son discours sur un ordre : «Suivés moy» (p.93).

Une fois dans le château, sa stature de chef s'affirme davantage encore : «Il veult achiever son entreprise»(p. 93). Il est celui qui donne les ordres : «Primes il fait clorre les trois portes et lever les pons, puis occirre le portier» (p. 93). Alors que Chrétien décrit l'action collective des trente et un chevaliers («Car cil ne se celerent plus/ Qui sor els sont venu lassus,/ Einz laissent corre les destriers », v. 1851-53), la prose se concentre sur les exploits du seul Alexandre, se contentant d'ajouter: "Pareillement sez homez se porterent et firent valloir.» Alexandre est ensuite attaqué par le traître Engrès qu'il désarçonne rapidement. Le comte, craignant pour sa vie, s'enfuit alors dans une haute tour en compagnie d'une troupe de chevaliers. Chez Chrétien, le combat contre le comte s'engage après que ce dernier a tué le Grec Calcedor à la grande tristesse d'Alexandre ${ }^{18}$, détail omis par le prosateur. Il donne lieu à une longue description qui insiste sur les qualités chevaleresques de l'adversaire du Grec :

Mes de grant force estoit li cuens

Et chevaliers estoit molt boens,

Q'au siecle nul meillor n'eüst

Se fel et traïtor ne fust. (v. 1879-83).

Pour le prosateur en revanche, il est hors de question de reconnaître la moindre qualité à un traître, comme on le verra encore.

Le comte espère pouvoir s'enfermer dans la tour en attendant les secours de la ville qui de fait s'organisent. Dans le Cligès en vers, un Grec plein de sagesse, Nabunal, suggère de barricader la porte d'accès à la forteresse. Son avis est suivi et les compagnons peuvent ensuite assaillir le comte et ses chevaliers qu'ils parviennent à maîtriser non sans de lourdes pertes dans leurs rangs. C'est à Alexandre qu'il revient, bien entendu, de terrasser le comte malgré la résistance acharnée de celui-ci ; mais le reste de l'opération présente à égalité les audacieux

${ }^{18}$ Pris de rage et de désespoir à la vue de son compagnon mort, Alexandre n'a qu'un désir: «Volentiers se il pooit / La mort son ami vencheroit. » (v. 1877-78). Le terme d' " ami » révèle la fraternité qui unit les jeunes Grecs. 
chevaliers qui pour la moitié paient de leur vie leur coup de main. Le prosateur pour sa part supprime le personnage de Nabunal : après avoir fait mourir un grand nombre d'ennemis, Alexandre revient à la porte de la première enceinte à laquelle frappent des hommes d'armes épuisés, ignorant ce qui se passe dans la forteresse. Un portier traitre propose alors en échange de sa vie de faire entrer par une petite poterne les soldats un à un, sans casque sous prétexte de vérifier leur identité. Il les conduit dans une salle où Alexandre et ses hommes les exécutent sans pitié. Le portier, traitre ou non au demeurant, est une figure assez familière dans le roman tardif. On le rencontre par exemple dans Ysaÿe le Triste, où il est régulièrement châtié avec sévérité par le nain Tronc ${ }^{19}$. On ne connaît pas le sort réservé à celui de Cligès, mais on peut imaginer qu'il obtiendra lui aussi la juste rétribution de sa traitrise.

C'est ensuite seulement qu'a lieu le combat d'Alexandre contre le comte. Le prosateur reprend à Chrétien l'arme dont le Grec frappe Engrès: une esparre (v. 2005) ou un tinel (p.95) mais présente le comte de manière bien moins avantageuse : Chrétien le montrait dos à un poteau, se défendant comme un animal aux abois ${ }^{20}$; dans la prose il se «musce contre un postel» (p. 95), puis, détail absent dans le texte en vers, il tourne autour du poteau pour échapper à son adversaire : pour le prosateur, le comte est plus une souris qu'un sanglier !

On le voit, il s'agit de montrer Alexandre non pas comme le primus inter pares qu'il est chez Chrétien, mais comme le chef incontesté d'une troupe de chevaliers à qui il impose ses décisions. Quant aux ennemis, ils doivent incarner les pires défauts : la traîtrise bien sûr, nous allons y revenir, mais aussi la lâcheté, l'arrogance, la faiblesse. Leur punition est à la mesure de la faute qu'ils commettent en osant contester la toute puissance d'Arthur. Car Alexandre n'est pas la seule figure de pouvoir dans l'épisode: Arthur s'avère un roi inflexible qui n'admet aucune résistance. Plusieurs scènes le montrent ainsi exerçant son autorité. À peine débarqué en Grande Bretagne, par exemple, il reçoit la soumission des paysans qui habitent aux alentours de Londres, puis des habitants de la ville qui se confondent en excuses et en explications et à qui il accorde sa merci avec magnanimité (p. 82). Comme l'a bien montré $\mathrm{M}$. Colombo Timelli21 ${ }^{21}$, cette séquence qui ne se trouve pas dans la version en vers n'est pas sans évoquer la rigueur avec laquelle le duc de Bourgogne Philippe le Bon contraint à la soumission les grandes villes de Flandre qui se sont révoltées contre lui, en particulier Gand. Le sort des quatre prisonniers d'Alexandre révèle également une transformation intéressante de la personnalité royale. Chrétien indique en effet que le jeune homme remet les prisonniers à la reine «par cortoisie» (v. 1341) mais aussi parce que « Ne volt que d'els eüst sesine/ $\mathrm{Li}$ rois, que tost les feïst pendre.» (v. 1344-45). Les Grecs approuvent la sagesse de cette décision, mais le roi, mécontent, convoque la reine. S'ensuit une discussion

\footnotetext{
${ }^{19}$ Voir Ysaÿe le Triste, Roman arthurien du moyen âge tardif, éd. A. Giacchetti, Rouen, Publications de l'Université de Rouen, 1989. Par exemple p. 52-54, les démêlés de Tronc et du portier de Menet et p. 364, la manière dont Tronc tue le portier de Val Douche qui lui a refusé l'entrée de la ville.

${ }^{20}$ «Et li cuens atout .I. hache/ Se fu mis delez .I. estache / Ou molt fierement se desfent, / Qui il consiut, parmi le fent. » (v. 1991-94).

${ }^{21}$ Voir «Le Cligès en prose (1455) ou l'actualisation d'un ancien conte en vers », art.cit. p. 340-41.
} 
qu'on imagine houleuse durant laquelle Alexandre et ses compagnons se reposent dans la chambre des dames. La reine revient au moment où Soredamor termine son long monologue intérieur sur le doux nom d'ami, de sorte qu'elle n'a pas le temps de s'adresser à Alexandre lequel est préoccupé par le sort des prisonniers. La reine décrit alors au jeune Grec le courroux du roi et confesse qu'elle a dû remettre les quatre hommes à Arthur. Le lendemain, le roi réunit son conseil pour décider du type de mort qui sera infligé aux prisonniers. Plusieurs chevaliers s'expriment, mais c'est l'avis du roi qui prévaut: les quatre hommes seront traînés. Le passage présente un roi qui finit par imposer sa décision mais non sans s'être heurté au préalable à la résistance de sa femme et de ses hommes. Ce n'est qu'au terme de délibérations que ses opposants se plient à sa volonté. Qu'en est-il dans le texte en prose? Alexandre remet les prisonniers à la reine sans explication. Le prosateur ajoute ensuite :

[V]ous devés savoir que toux ceulx de l'ost prisoient Alixandre et le looient, excepté le roy, qui ne disoit mot a cause qu'il estoit marri de ce que il ne avoit lez prisonniers pour lez faire mourir. Il lez requist a la reyne, laquelle lui les acorda. (p. 84).

L'éloge dont tout le monde comble Alexandre ne concerne plus la sagesse dont il a fait preuve en remettant les prisonniers à la reine, mais plus largement la conduite qui a été la sienne pendant le combat. Le roi pour sa part manifeste son mécontentement en réservant son admiration. Mais il obtient vite ce qu'il veut : il lui suffit d'exiger les prisonniers de la reine pour que celle-ci les lui remette sans discussion aucune ${ }^{22}$. Enfin, le roi ne juge pas nécessaire de demander l'avis de ses sujets sur le châtiment à infliger aux traîtres :

[L]e roi fait devant soi venir les IIII prisonniers, et adjuge qu'ilz soient atachiés aux queues de chevaulx par piés et par mains, et qu'en ce point ilz soient detraynés tant que mort s'en ensuive. (p. 86).

Personne n'ose contester la volonté du roi par crainte de s'attirer les foudres de celui-ci et bien que les traîtres meurent de la même manière dans le texte de Chrétien et dans la version bourguignonne, la prose offre toutefois davantage de détails réalistes et horribles :

Li rois entor le chastel fait

Traïner a quatre chevaux

Les traïtors parmi les vaus

Et par tertres et par larriz. (v. 1492-1495).

\footnotetext{
${ }^{22} \mathrm{Ce}$ qui a pour conséquence une modification un peu incongrue : la reine est présente dans la chambre des dames quand Soredamor se demande avec inquiétude comment s'adresser à Alexandre. Ce n'est donc pas l'arrivée de la reine qui l'empêche de mettre son plan à exécution. Alexandre, semble-t-il, se lasse : "Tandis que Soredamours estoit en ce penser, Alixandre ala veioir le roy...» (p. 85)!
} 
Le bourreau les ampoigne, puis les atache comme dessus est dit. Et, a mesures qu'ils sont loiés, il chasse lez chevaulx au loingz; si s'en vont courant ci lez ungz la les aultres par montaignes, rociers, chardons, ronces, ortiez, espines, tant que lez IIII meschans hommes furent executéz a mort et qu'ilz furent tellement deffigurés qu'il ne leur demoura membre nul entier. (p. 86).

L'apparition du bourreau, exécuteur officiel de la justice royale, souligne le caractère institutionnel de la peine réservée aux prisonniers ${ }^{23}$. Le roi s'entoure d'officiers chargés de mettre en œuvre les décisions qu'il a prises de manière autoritaire.

De même, à la fin de l'épisode, le supplice du comte Engrès est explicité dans la prose. Chrétien se contente de dire que «li rois n'a plus atendu/ Que lors n'en face la joustice.» (v. 2166-67), mais le prosateur précise :

Alixandre livra son prisonnier au roy, qui trencier lui fist la teste et escarteller, puis fichier ladicte teste ou bout d'une lance ou millieu de la premiere porte. (p. 97).

Si le châtiment des traîtres est décrit avec plus de complaisance dans la version en prose que dans le texte en vers, cela tient sans doute à l'analogie entre la situation décrite dans le roman et la réalité politique en Bourgogne au $\mathrm{XV}^{\mathrm{e}}$ siècle. Confronté à de nombreuses rébellions, Philippe le Bon avait tout intérêt à les présenter comme autant de trahisons qu'il pouvait réprimer avec la plus extrême rigueur. En 1437 par exemple, des émeutes éclatèrent à Bruges et dégénérèrent en une véritable guerre civile qui embrasa la Flandre. En 1438, Bruges se soumit finalement au duc qui imposa de lourdes pénalités à la ville. Entre autres, les quarante personnes les plus compromises dans les événements furent décapitées et leurs têtes exposées aux portes de la ville ${ }^{24}$. Le Cligès en prose, composé dans l'entourage du duc de Bourgogne, reflète la sévérité avec laquelle était jugée toute résistance au pouvoir du prince.

Chrétien certes condamne lui aussi la trahison commise par le comte et Arthur reproche du reste à ses barons de lui avoir conseillé de confier sa terre à Engrès :
Quant li rois ot ceste novele,
Trestouz ses barons en apele,
Iriez et pleins de maltalent.
Por ce que melz les entalent
De confondre le traitor,
Dist que touz li blames est lor
De son tribol et de sa guerre,

${ }^{23}$ Sur les débuts de la fonction de bourreau et les tâches qui incombe au personnage, voir l'article de Valérie Toureille dans Le Dictionnaire du Moyen Age, sous la direction de Claude Gauvard, Alain de Libera et Michel Zink, Paris, Puf, 2002.

${ }^{24}$ Sur ce point, voir Bertrand Schnerb, L'État bourguignon, Paris, Perrin, 1999, 2005 pour l'édition de poche, chap. 22 : L'état bourguignon face au particularisme urbain (p. 379 pour le cas de Bruges, mais voir aussi la guerre de Gand). 
Car par els lessa il sa terre

Et mist en la main au felon

Qui pires iert de Ganelon (v. 1063-72).

Comparé à Ganelon, dont il subira le châtiment, Engrès achète l'amitié de ses hommes en répartissant les biens qu'il a pillés dans la ville de Londres. Prévoyant la colère d'Arthur, il a passé tout l'été à renforcer les défenses de la forteresse de Guinesores qu'il rejoint de nuit dès l'annonce du débarquement du roi. Le terme de traïtor revient à plusieurs reprises au début de l'épisode pour désigner le comte ${ }^{25}$. Les quatre chevaliers faits prisonniers par Alexandre sont, on l'a vu, également qualifiés de traîtres et punis comme tels ${ }^{26}$. Au moment où les assiégés préméditent une sortie de nuit, Chrétien intervient encore une fois pour stigmatiser la trahison. Alors que la nuit était jusqu'à là obscure, la lune se met soudain à luire précisément quand les chevaliers armés et casqués sortent en silence, de sorte que le guet les aperçoit et peut donner l'alerte. Chrétien suggère que c'est là la volonté de Dieu :

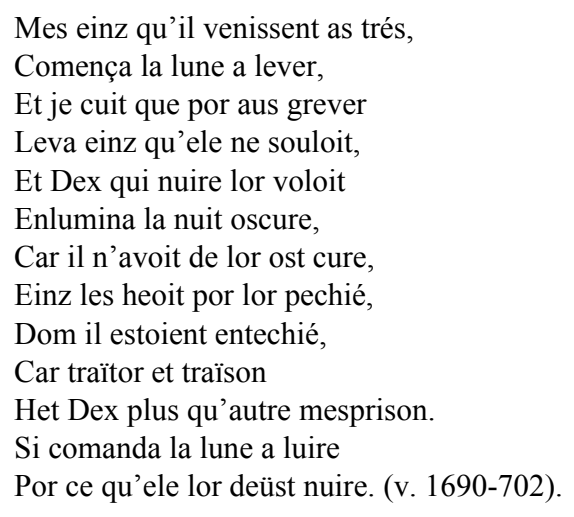

Dieu pourrait bien avoir avancé quelque peu le phénomène naturel du lever de la lune pour montrer combien il désapprouve l'entreprise des traîtres. Mais c'est Chrétien (je cuit) qui prend en charge l'interprétation de cet accident malheureux, préservant ainsi une certaine ambiguïté. Peut-être Dieu s'est-il contenté de dissiper les nuages qui rendaient la nuit obscure. Le prosateur est plus affirmatif: il s'agit bel et bien d'un miracle. Bouleversant l'ordre de la nature, le jour se lève bien plus tôt qu'à l'accoutumée :

[P]ar le commandement de Dieu qui nuist selon justice aux pecheurz, le ciel fu enluminé bien heure et demie plus tost que le courz de nature n'avoit acoustumé de faire. (p. 89).

\footnotetext{
${ }^{25} \mathrm{~V} .1067,1083,1220,1228,1237$

${ }^{26}$ V. 1348 « retez de traïson»; v. 1363 : traïtors ; id. v. 1431, 1435, 1494.
} 
De manière générale, la prose amplifie considérablement les accusations de trahison : elle en précise la teneur et met en scène la trahison de manière beaucoup plus frappante que le texte de Chrétien.

On note d'abord que les termes utilisés pour désigner le comte et ses hommes sont plus nombreux et plus divers. Le comte est un traître, un malfaiteur, et surtout un rebelle ${ }^{27}$, comme Arthur le déclare à ses barons :

Il leur dist qu'ilz ont meffait de lui avoir consillié qu'il laissat sa terre a ung rebelle, faulx et trahitour malvés, qui veult seignourier ad ce dont il n'est pas digne d'estre varlet. (p. 80).

Le comte n'est pas coupable d'avoir mal servi son maître, de lui avoir été infidèle. Il a voulu prendre sa place, faute gravissime. Ceux qui l'assistent sont ses «complices» (p. 86, p. 89). Alors que Chrétien appelle en général les assiégés «cil dou chastel », la prose les désigne plus souvent comme traîtres ${ }^{28}$.

Par ailleurs plusieurs ajouts révèlent la gravité avec laquelle le prosateur traite le sujet de la trahison. L'anecdote du portier qui pour obtenir son salut trompe ceux de son camp montre la bassesse des hommes qui entourent le comte. Traître, Engrès ne peut réunir que des hommes de son espèce qui le trahiront au bout du compte. Par ailleurs, l'attitude d'Alexandre face aux ennemis, comme celle d'Arthur, est beaucoup plus sévère dans la version en prose que dans la version en vers. On a déjà vu qu'une fois dans la forteresse, il fait tuer le premier portier et jeter son corps dans les fossés. Lorsque le comte trouve refuge dans la tour, ses hommes restés au-dehors se mettent à la merci d'Alexandre qui les conduit sur les murailles et les oblige à en sauter (p. 93) ${ }^{29}$, opération qu'il renouvelle avec vingt autres soldats qui se sont pourtant eux aussi rendus à lui :

Alixandre prent leurz espees, puis lez fait monter aux creniaux, et comme il a fait aux aultres pour le guerredon de leur rebellion et malivolence il les fait saulter és fossés, esquelz ylz meurent miserablement. (p. 94).

Il condamne à la même exécution sommaire une troisième fournée d'ennemis (p. 96) et n'épargne que le comte afin de pouvoir le livrer à Arthur.

Chez Chrétien au contraire, Alexandre fait preuve de générosité envers les comparses d'Engrès. Loin de les tuer, il les encourage à aller se rendre à Arthur et se porte garant de la bienveillance du roi :

« Issiez tuit desarmé la fors

Encontre mon seignor le roi

Et si li dites de par moi

${ }^{27}$ Les termes rebeller, rebellion, rebelle apparaissent quatre fois dans le paragraphe 11. Voir aussi paragraphe 15, p. 83 .

${ }^{28}$ Voir par ex. v. 1261 et p. 83 : le prosateur qui a d'abord suivi Chrétien («Bien cuident estre asseur ceulx du chasteau »), se corrige en apelant les ennemis « les trahiteurs ».

${ }^{29}$ Ce détail avait déjà été relevé par C. C. Willard dans «The Misfortunes of Cligès », art.cit. p. 401. 
Qu'Alixandres vos i envoie.

Ne perdroiz mie vostre voie,

Car tout son maltalent et s'ire

Vos pardonra li rois mes sire,

Tant est il frans et deboennaire. » (v. 2144-51).

Seul le comte, selon l'Alexandre de Chrétien, mérite la mort. De fait, il le livre à Arthur qui le fait exécuter. Dans la version en prose, l'exécution du comte est précédée d'un premier châtiment infligé par Alexandre lui-même. Après avoir assisté au supplice de tous ses hommes, le comte est conduit à son tour sur les murailles où, dévêtu et garrotté, il est exposé aux regards des habitants de la ville ${ }^{30}$. Il se lamente alors sur sa destinée, blâme Fortune et, se croyant abandonné de Dieu, succombe au désespoir, commettant ainsi un ultime péché. Comme il supplie Alexandre de lui donner la mort pour lui épargner la fin misérable que lui réserve le roi, celui-ci accompagne son refus d'une sévère condamnation morale qui s'achève sur la terrible promesse suivante :

«Or regarde dont de com grande affliction et martire ta charongne doit estre tourmentee pour la recompensacion de ton delict et de celle grant perte de monde que tu avoies seduit, enchanté et suborné par tes faulces parolles. » (p. 97).

La cruelle mise en scène d'Alexandre, comme l'exposition de la tête décapitée du comte, a pour objet de frapper la population et de graver dans les esprits le sort qui attend les rebelles. Si la seconde évoque, on l'a dit, le traitement que le duc de Bourgogne lui-même a réservé aux chefs des révoltes urbaines, la première a son équivalent dans des romans contemporains. Ysä̈e le Triste par exemple multiplie les scènes macabres dont le nain Tronc est en général l'orchestrateur. C'est ainsi que pour punir le traître Helyas, Tronc imagine de lui couper tour à tour les oreilles, les doigts et le nez, avant de le décapiter, en compagnie de ses acolytes, et de pendre les corps et les têtes à un arbre près de leur château ${ }^{31}$. Helyas a beau supplier Tronc d'abréger ses souffrances, celui-ci, comme Alexandre face à Engrès, lui rétorque qu'il lui faudra acheter la mort beaucoup plus cher encore :

« Tu ne le (= la mort) fais encore que barguignier, et sy en aras peu offert a vespre ; se tu l'achattes trop, tant le devras tu mieulx avoir. ${ }^{32}$

L'horreur que suscitent ce spectacle et d'autres similaires ${ }^{33}$ est rapportée à maintes reprises. Sur un mode plus humoristique, mais finalement tout aussi horrible, les dames que Saintré appelle à juger la trahison de Belle Cousine

\footnotetext{
${ }^{30}$ Alors qu'ils sont en train de s'apitoyer sur le sort de leur comte, les troupes d'Arthur qui arrivent en profitent pour les décimer (p. 97)!

${ }^{31}$ Ysä̈e le Triste, Roman arthurien du moyen âge tardif, éd. cit., p. 482

${ }^{32} \mathrm{Id}$.

${ }^{33}$ Voir dans Ysaÿe le Triste, p. 348, le sort de trois chevaliers tombés aux mains d'Englier et de Tronc.
} 
imaginent des châtiments spectaculaires à valeur d'exemple. Madame du Perche propose que la dame déloyale soit «toute nue despouillee et de la chainture contremont, et toute reze, puis oingte de bon miel et menee par la ville, laissant la mengier aux mouches $\|^{34}$. La traitrise, en amour comme en politique, est ainsi punie avec la plus extrême sévérité et une sévérité théâtralisée d'une manière nouvelle. La tirade amère que le comte Engrès prononce du haut des murailles de sa forteresse et la réponse intransigeante d'Alexandre s'inscrivent parfaitement dans ce goût de la mise en scène du châtiment.

Ainsi se dégage de la prose l'image d'un chef de guerre autoritaire, qui domine ses hommes aussi bien que ses ennemis. Alexandre, destiné à être empereur de Constantinople, comme le rappelle le titre de la mise en prose: Le Livre de Alixandre empereur de Constentinoble et de Cligés son filz, a bien l'étoffe d'un prince puissant. Quant à Arthur, le roi parfois contesté de Chrétien, il apparaît comme un maître craint et obéi qui exerce avec autorité toutes ses prérogatives, en particulier dans le domaine de la justice. Le prosateur infléchit donc avec une remarquable cohérence le roman de Chrétien. Comme l'a bien montré J. Taylor, il ne s'agit pas nécessairement dans son esprit de l'investir d'un nouveau sens politique, mais plutôt de le rendre compréhensible à son public. Bien que d'un tout autre ordre en apparence, les modifications sont finalement comparables au travail de «traduction» auquel il se livre par ailleurs. Son public ne comprend pas l'ancien langaige françoys. Il ne peut pas concevoir non plus qu'un prince soit autre chose qu'un maitre tout puissant, concentrant tout le pouvoir. Le prosateur transpose pour lui le système politique tel qu'il apparait chez Chrétien, tout comme les auteurs des romans antiques avant lui ont adapté les fables anciennes à la réalité idéologique du $\mathrm{XII}^{\mathrm{e}}$ siècle. Si ce que nous appelons «anachronisme», faute de bien comprendre la fonction et la pertinence des modifications, est moins frappant dans le travail du prosateur bourguignon, puisque la différence culturelle entre l'époque de Chrétien et la sienne est moindre que celle qui sépare les auteurs de romans antiques de leurs modèles, c'est pourtant bien le même phénomène d'«acculturation » qui est en jeu.

Michelle Szkilnik

Paris III

\footnotetext{
${ }^{34}$ Jehan de Saintré, éd. J. Blanchard et trad. M. Quereuil, Paris, Livre de poche (Lettres gothiques), 1995, p. 526.
} 